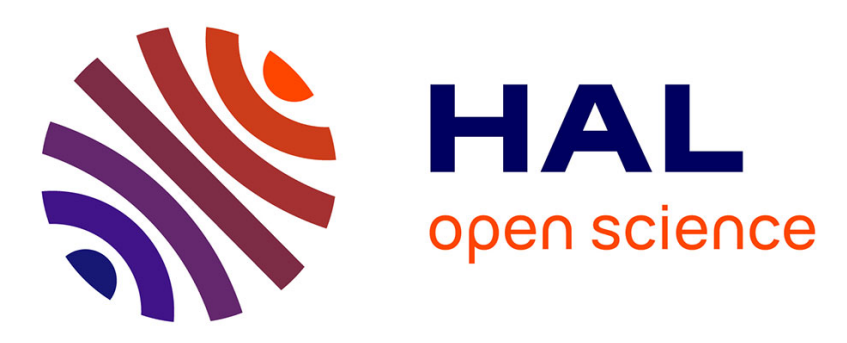

\title{
The Fundamental Diagram on the Ring Geometry for Particle Processes with Acceleration/Braking Asymmetry
}

Cyril Furtlehner, Jean-Marc Lasgouttes, Maxim Samsonov

\section{- To cite this version:}

Cyril Furtlehner, Jean-Marc Lasgouttes, Maxim Samsonov. The Fundamental Diagram on the Ring Geometry for Particle Processes with Acceleration/Braking Asymmetry. TGF'11 - Traffic and Granular Flow, Sep 2011, Moscou, Russia. hal-00646988

\section{HAL Id: hal-00646988 \\ https://hal.inria.fr/hal-00646988}

Submitted on 1 Dec 2011

HAL is a multi-disciplinary open access archive for the deposit and dissemination of scientific research documents, whether they are published or not. The documents may come from teaching and research institutions in France or abroad, or from public or private research centers.
L'archive ouverte pluridisciplinaire HAL, est destinée au dépôt et à la diffusion de documents scientifiques de niveau recherche, publiés ou non, émanant des établissements d'enseignement et de recherche français ou étrangers, des laboratoires publics ou privés. 


\title{
The Fundamental Diagram on the Ring Geometry for Particle Processes with Acceleration/Braking Asymmetry
}

\author{
Cyril Furtlehner ${ }^{1}$, Jean-Marc Lasgouttes ${ }^{2}$, and Maxim Samsonov ${ }^{1}$ \\ 1 INRIA Saclay - LRI, Bat. 490, Université Paris-Sud - 91405 Orsay cedex (France) \\ cyril.furtlehner@inria.fr \\ 2 INRIA Paris-Rocquencourt - Domaine de Voluceau B.P. 105 - 78153 Le Chesnay \\ cedex (France) jean-marc.lasgouttes@inria.fr
}

Summary. The slow-to-start mechanism is known to play an important role in the particular shape of the fundamental diagram of traffic and to be associated to hysteresis effects of traffic flow. We study this question in the context of stochastic processes, namely exclusion and queueing processes, by including explicitly an asymmetry between deceleration and acceleration in their formulation. Spatial condensation phenomena and metastability are observed, depending on the level of the aforementioned asymmetry. The relationship between these 2 families of models is analyzed on the ring geometry, to yield a large deviations formulation of the fundamental diagram (FD).

\section{Introduction}

In the microscopic theory of traffic [1, 2], an asymmetry between acceleration and deceleration, observed for example in the headway distribution, is empirically known to be responsible of the way spontaneous congestion occurs, as can be seen experimentally on a ring geometry for example [3]. This is often referred as the slow-to-start mechanism, not present in the original cellular automaton of Nagel-Schreckenberg [4], but added in refined versions like the "velocity dependent randomized" one (VDR) [5], which exhibits a first order phase transition between the fluid and the congested phases and hysteresis phenomena [6] associated to metastable states. This is partly reflected in the shape of the FD. In this work, we analyze this property in the context of stochastic processes. We propose a minimal extension of TASEP to include that mechanism, and a way to compute the corresponding FD on the ring geometry, where

- $\quad$ some equivalence between particle systems and queueing processes holds, either exactly or with good approximation, and can be exploited to compute the FD;

- the relation between the density of vehicles and the flux is a well defined quantity in the thermodynamic limit on this geometry, since the number of cars is conserved (canonical ensemble). 
The paper is organized as follows: in Section 2, we define a family of exclusion processes relevant to traffic and we discuss how these can be reformulated in terms of a tandem queue process with a dynamically coupled stochastic service rate. The invariant measure of such a process at steady-state is determined in Section 3. Finally, in Section 4, we discuss the computation of the FD in the canonical ensemble (with a fixed number of cars), when a product form of the equivalent queueing process holds.

\section{Multi-speed exclusion processes}

\subsection{Model definition}

In the totally asymmetric version of the exclusion process (TASEP), particles move randomly on a 1-d lattice, always in the same direction, hopping from one site to the next at times following a Poisson process and conditionally that the next site is vacant. In the Nagel-Schreckenberg cellular automaton, the dynamics is parallel and the vehicles' speeds are encoded in the number of slots that they can cover in one jump. This speed can adapt stochastically, depending on the available space in front of the particle. We propose to combine the braking and accelerating features of the Nagel-Schreckenberg models, with the locality of the simple ASEP model, in which only two consecutive sites do interact at a given time. For this, we allow each particle to change stochastically its hopping rate, depending on the state of the next site. For a 2 -speed model, let $A$ (resp. $B$ ) denote a site occupied by a fast (resp. slow) vehicle, and let $O$ denote empty sites. The model is defined by the following set of reactions, involving pairs of neighboring sites:

$$
\begin{array}{ll}
A O \stackrel{\mu_{a}}{\longrightarrow} O A \quad \text { simple move of fast vehicle, } \\
B O \stackrel{\mu_{b}}{\longrightarrow} O B \quad \text { simple move of slow vehicle, } \\
B O \stackrel{\gamma}{\longrightarrow} A O \quad \text { slow vehicle spontaneously accelerates, } \\
A \bar{O} \stackrel{\delta}{\longrightarrow} B \bar{O} \quad \text { fast vehicle brakes behind another one }(\bar{O}=A \text { or } B) .
\end{array}
$$

Here $\mu_{a}, \mu_{b}, \gamma$ and $\delta$ denote the transition rates. The first couple of transitions corresponds to normal movements of vehicles. The other two encode the fact that a slow vehicle tends to accelerate when there is space ahead (3), while in the opposite case (4), it tends slow down. The asymmetry between braking and acceleration is explicitly present in the model with $\gamma$ different from $\delta$. Our model can be viewed as the sequential version of the Appert-Santen model [7], in which there is one single speed, but particles have 2 states (at rest and moving). In the remainder of the paper, we will consider this model on the ring geometry.

Let us first notice that this model contains and generalizes several sub-models which are known to be integrable with particular rates. The hopping part $(1,2)$ is just the totally asymmetric exclusion process $[8,9]$ (TASEP) when $\mu_{a}=\mu_{b}$, which is known to be integrable. The acceleration/deceleration dynamics is equivalent to the coagulation/decoagulation models, which are known to be solvable by the empty interval method and by free fermions for particular sets of rates [10], but the whole process is presumably not integrable. 


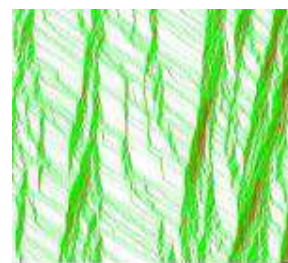

(a)

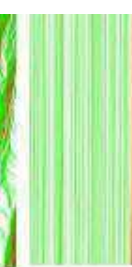

(b)

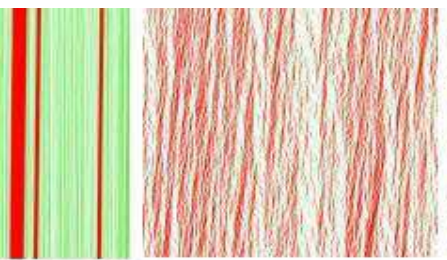

(c)

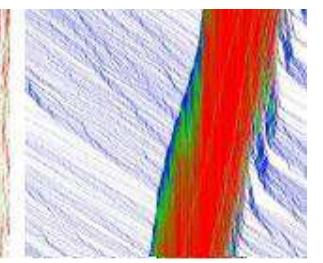

(d)

Fig. 1. Space-time diagram for multi-speed exclusion process with 2 speed levels (a)-(c) and 3 in (d). Time is going downward and particles to the right. Red, green and blue represent different speeds in increasing order. The size of the system is 3000 except for (b) where it is 100000 . Setting are $\mu_{a}=100, \mu_{b}=10, \gamma=100, \delta=2$ for (a) and (b) and $\delta=10$ for (c), all with density $d=0.2$.

\subsection{Relation to tandem queues}

In some cases, the model can be exactly reformulated in terms of generalized queueing processes (or zero range processes in the statistical physics parlance), where the service rate of each server follows itself a stochastic dynamics [11]. In this preceding work, however, we considered exclusion processes involving three consecutive sites interactions. In fact, in the present case, a large family of sub-models can be mapped onto such queueing processes. The mapping works only on the ring geometry, by identifying servers either with

(i) cars: clients are the empty sites;

(ii) empty sites: clients are the vehicles.

In our case, the mapping of type (i) is exact. In the corresponding queueing process, servers are associated either with fast or slow cars, having then service rates $\mu_{a}$ or $\mu_{b}$. Slow queues become fast at rate $\gamma$, conditionally to having at least one client, while empty fast queues become slow at rate $\delta$.

The mapping of type (ii) is more informative with respect to jam distribution [11], but is not possible with transitions limited to 2-consecutive sites interactions, because in that case homogeneity is not maintained in the clusters, and information beyond the number of cars and the rate of the car leaving the queue is needed to know the service rate of the queue. Nevertheless, to be able to get meaningful information on the jam structure, i.e. on the long range correlations of the model associated to cluster formations, this is the mapping that we will consider later in Section 3.

\subsection{Numerical observations}

Some numerical observations are illustrated on Figure 1. When no asymmetry between braking and accelerating is present $(\gamma=\delta)$, as in TASEP on a ring, no spontaneous jam formation occurs. As the density $d$ of cars increases, one observes a smooth transition between a TASEP of fast particles for small $d$ to a TASEP of slow particles around $d \simeq 1$. When the ratio $\gamma / \delta$ is reduced, there is a proliferation of small jams associated with slow vehicles as seen on Figure 1.c. Instead when it increases slow car are less present as well as small jams as shown on Figure 1.a. Above some threshold 
of this ratio $\gamma / \delta$, which depends on the density, a condensation phenomenon occurs as seen on Figure 1.b; one or more large jams contain a finite fraction the total number of cars. It is therefore tempting to understand this phase separation with the ZRP interpretation [12] of a spatial condensation phenomena in the canonical ensemble [13]. In this viewpoint, we expect a condensation mechanism to occur if the apparition of slow vehicles is a sufficiently rare event [11]. In our context, the probability that a vehicle joining a cluster of particles is still of type $A$ when it reaches the front is decaying exponentially

$$
p\left(\mu=\mu_{a}\right)=e^{-\delta t(s)}=p_{s},
$$

with $t(s)$ the waiting time in a queue of size $s$. Assuming stationarity and the possible acceleration of the front car, we obtain self-consistently

$$
t(s)=s\left[\frac{1}{\mu_{a}} p_{s}+\frac{\mu_{a}+\gamma}{\mu_{a}\left(\mu_{b}+\gamma\right)}\left(1-p_{s}\right)\right] .
$$

This observation will be made more precise in Section 3. It implies qualitatively that starting with a distribution of jams with different effective service rates, as time evolves, long-lived jams with effective service rates slightly above $\mu_{b}+\gamma\left(\mu_{a}-\right.$ $\left.\mu_{b}\right) /\left(\mu_{a}+\gamma\right)$ are able to survive. If the situation with a single jam is not stable, which is observed below some threshold density, then no large jam may develop at all, and only small fluctuations are to be observed. Typically this occurs when $\delta \ll \mu_{b}$ and $\delta<\gamma$ (Figure 1(b)). In contrary when $\delta>\gamma$ one likely observes the kind of jams in Figure 1(c).

We have also simulated a model with 3 speed levels, see Figure 1(d). In that case, small jams may have different speeds, depending on which type of slow car is leading. Then a cascade mechanism takes place, slow speed regions generate even slower speed clusters of cars and so on, and some kind of synchronized flow is observed.

\section{Queues with two-state service rates}

The mapping of type (ii) defined in section 2.2, while not exact here, may help us to capture the correlations associated to cluster formations. To this end, consider first the dynamics and steady state regime of a single cluster of vehicles of size $n_{t}$ and assume that vehicles of type $A$ [resp. $B]$ join the queue with rate $\lambda_{a}\left[\right.$ resp. $\left.\lambda_{b}\right]$, while they leave the queue with rate $\mu_{a}$ [resp. $\mu_{b}$ ]. $\lambda \stackrel{\text { def }}{=} \lambda_{a}+\lambda_{b}$ represents the intensity of the incoming process, and particles decelerate with rate $\delta$ in the bulk of the queue. At position $n$ in the queue, counting from the back end position, the probability for a particle to be still of type $A$ is then

$$
p_{n} \stackrel{\text { def }}{=} \frac{\lambda_{a}}{\lambda} r^{n} \quad \text { with } \quad r \stackrel{\text { def }}{=} \frac{\lambda}{\lambda+\delta} .
$$

Since the front end interface of the cluster has no causal effect on the rest of the queue, except on the front vehicle which may accelerate with rate $\gamma$, we can consider the dynamics of the sequence independently of the motion of the front interface. Under the additional assumption of independence between speed labels in the bulk of the queue, we consider the joint probability $P_{t}(n, \tau)=\mathbb{P}\left(n_{t}=n, \mu_{t}=\mu_{a} \tau+\mu_{b} \bar{\tau}\right)$, 
i.e. the joint probability that the queue has $n$ clients and its front car is of type $A$ $(\tau=1)$ or $B(\bar{\tau} \stackrel{\text { def }}{=} 1-\tau=1)$. With $p_{n}(\tau) \stackrel{\text { def }}{=} p_{n} \tau+\left(1-p_{n}\right) \bar{\tau}$, the master equation then reads

$$
\begin{aligned}
\frac{d P_{t}(n, \tau)}{d t} & =\lambda\left(P_{t}(n-1, \tau)-P_{t}(n, \tau)\right)+\left(\mu_{a} P_{t}(n+1,1)+\mu_{b} P_{t}(n+1,0)\right) p_{n}(\tau) \\
& -\left(\mu_{a} \tau+\mu_{b} \bar{\tau}\right) P_{t}(n, \tau)+\gamma(\tau-\bar{\tau}) P_{t}(n, 0), \quad n \geq 2 \\
\frac{d P_{t}(1, \tau)}{d t} & =\left(\lambda_{a} \tau+\lambda_{b} \bar{\tau}\right) P_{t}(0)-\lambda P_{t}(1, \tau)+\left(\mu_{a} P_{t}(2,1)+\mu_{b} P_{t}(2,0)\right) p_{1}(\tau) \\
& -\left(\mu_{a} \tau+\mu_{b} \bar{\tau}\right) P_{t}(1, \tau)+\gamma(\tau-\bar{\tau}) P_{t}(1,0), \\
\frac{d P_{t}(0)}{d t} & =-\lambda P_{t}(0)+\mu_{a} P_{t}(1,1)+\mu_{b} P_{t}(1,0) .
\end{aligned}
$$

It is a special case of a queueing process with a 2-level dynamically coupled stochastic service rate generalizing queueing processes with stochastic service $[14,11$, 10]. In the stationary regime, we denote

$$
\pi_{n}^{a} \stackrel{\text { def }}{=} P\left(n, \mu=\mu_{a}\right), \quad \pi_{n}^{b} \stackrel{\text { def }}{=} P\left(n, \mu=\mu_{b}\right),
$$

and $\pi_{0}=P(n=0)$. Consider the generating functions

$$
g_{a, b}(z) \stackrel{\text { def }}{=} \sum_{n=1}^{\infty} \pi_{n}^{a, b} z^{n} \quad \text { and } \quad g(z) \stackrel{\text { def }}{=} \pi_{0}+g_{a}(z)+g_{b}(z)
$$

and let

$$
\begin{aligned}
& \lambda^{2} u \stackrel{\text { def }}{=} \lambda_{a}\left(\mu_{a}-\mu_{b}\right) \quad \lambda^{2} w \stackrel{\text { def }}{=}\left(\mu_{a} \mu_{b}+\lambda_{a} \mu_{a}+\lambda_{b} \mu_{b}+\gamma \mu_{a}\right) \pi_{0} \\
& \lambda^{2} v \stackrel{\text { def }}{=}\left(\lambda_{a} \mu_{b}+\lambda_{b} \mu_{a}\right) \pi_{0} \quad \Delta \stackrel{\text { def }}{=}\left(\lambda-\gamma+\mu_{a}-\mu_{b}\right)^{2}+4 \lambda \gamma .
\end{aligned}
$$

The invariant measure is obtained as follows [15]:

Theorem 3.1 (i) $g(z)$ satisfies the functional equation of the type

$$
u g(r z)=v z+w-\left(z-z^{+}\right)\left(z-z^{-}\right) g(z),
$$

where $z^{ \pm}$are given by

$$
z^{ \pm}=\frac{1}{2 \lambda}\left(\mu_{a}+\mu_{b}+\lambda+\gamma \pm \sqrt{\Delta}\right) .
$$

(ii) The solution reads:

$$
g(z)=\sum_{n=0}^{\infty}(-u)^{n} \frac{v r^{n} z+w}{\prod_{k=0}^{n+1}\left(z r^{k}-z^{+}\right)\left(z r^{k}-z^{-}\right)} .
$$

Using the following partial balance relation,

$$
\lambda \pi_{n}=\mu_{a} \pi_{n+1}^{a}+\mu_{b} \pi_{n+1}^{b}, \quad \forall n \geq 0,
$$


the reduced generating functions $g_{a, b}$ are then obtained as

$$
g_{a}(z)=\frac{\mu_{b}}{\mu_{a}-\mu_{b}} \pi_{0}+\frac{\lambda z-\mu_{b}}{\mu_{a}-\mu_{b}} g(z), \quad g_{b}(z)=\frac{\mu_{a}}{\mu_{b}-\mu_{a}} \pi_{0}+\frac{\lambda z-\mu_{a}}{\mu_{b}-\mu_{a}} g(z) .
$$

Upon using Cauchy integrals, the $\pi_{n}^{a, b}$ are then given as sums of geometric laws. From the radius of convergence $z^{-}$of $g$, the limit of ergodicity is obtained for $z^{-} \geq 1$, i.e. for

$$
\lambda \leq \mu_{b}+\gamma \frac{\mu_{a}-\mu_{b}}{\mu_{a}+\gamma}
$$

\section{Computing the fundamental diagram of product measures}

\subsection{Fundamental diagram}

In practice, points plotted in experimental FD studies are obtained by averaging data from static loop detectors over a few minutes (see e.g. [2]), but spatial average is much easier to obtain analytically. The equivalence between time and space averaging is not an obvious assumption, but since jams are moving, space and time correlations are combined in some way [16] and we consider this assumption to be quite safe. In what follows, we will therefore compute the FD along with its fluctuations, by considering the conditional probability measure $P_{\lambda}(\phi \mid d)$ for a closed system, where

$$
\left\{\begin{array} { l l } 
{ d = \frac { N } { N + L } , } \\
{ \phi = \frac { \Phi } { N + L } , }
\end{array} \quad \text { with } \left\{\begin{array}{ll}
L & \text { number of queues } \\
N=\sum_{i=1}^{L} n_{i} & \text { number of vehicles } \\
\Phi=\sum_{i=1}^{L} \mu_{i} \mathbb{1}_{\left\{n_{i}>0\right\}} & \text { integrated flow }
\end{array}\right.\right.
$$

are spatial averaged quantities and represent respectively the density and the traffic flow. $N$ and $L$ are fixed, which means that we are working with the canonical ensemble. If we assume that we are in the conditions of having a product form [17] (see [15] for details in this context) for the stationary distribution with individual probabilities $\pi^{\lambda}(n, \mu)$ associated to each queue taken in isolation, then, taking into account the constraints yields the following form of the joint probability measure:

$$
P\left(\left\{n_{i}, \mu_{i}\right\}\right)=\frac{\delta\left(N-\sum_{i=1}^{L} n_{i}\right)}{Z_{L}[N]} \prod_{i=1}^{L} \pi^{\lambda}\left(n_{i}, \mu_{i}\right),
$$

with the canonical partition function

$$
Z_{L}[N] \stackrel{\text { def }}{=} \sum_{\left\{n_{i}, \mu_{i}\right\}} \delta\left(N-\sum_{i=1}^{L} n_{i}\right) \prod_{i=1}^{L} \pi^{\lambda}\left(n_{i}, \mu_{i}\right)
$$

where $\delta$ denotes now the usual Dirac function. When this form is not exact but constitutes a mean-field ansatz, some flow conservation conditions have to be imposed, which for the model under consideration concern $\lambda$ and $\lambda_{a}$ and read 
Fundamental Diagram on the Ring with Acceleration/Braking Asymmetry

$$
\lambda_{a}=\mu_{a} \sum_{n=1}^{\infty} \pi_{n}^{a}, \quad \lambda_{b}=\mu_{b} \sum_{n=1}^{\infty} \pi_{n}^{b}=\lambda-\lambda_{a}
$$

The density-flow conditional probability distribution takes the form

$$
P(\phi \mid d)=\frac{Z_{L}[N, \Phi]}{Z_{L}[N]}
$$

with

$$
Z_{L}[N, \Phi] \stackrel{\text { def }}{=} \sum_{\left\{n_{i}, \mu_{i}\right\}} \delta\left(N-\sum_{i=1}^{L} n_{i}\right) \delta\left(\Phi-\sum_{i=1}^{L} \mu_{i} \mathbb{1}_{\left\{n_{i}>0\right\}}\right) \prod_{i=1}^{L} \pi^{\lambda}\left(n_{i}, \mu_{i}\right) .
$$

$Z_{L}[N]$ and $Z_{L}[N, \Phi]$ represent respectively the probability of having $N$ vehicles and the joint probability for having at the same time $N$ vehicles and a flux $\Phi$, under the unconstrained product form. Under this product form, we expect $d$ and $\phi$ to satisfy a large deviations principle (see e.g. [18]) i.e. that there exist two rate functions $I(d)$ and $J(d, \phi)$ such that, for large $L$,

$$
Z_{L}(N) \asymp e^{-L I(d)}, \quad Z_{L}[N, \Phi] \asymp e^{-L J(d, \phi)} .
$$

In other words, we expect a large deviations version of the fundamental diagram of the form

$$
P(\phi \mid d) \asymp e^{-L K(\phi \mid d)}, \quad \text { with } \quad K(\phi \mid d) \stackrel{\text { def }}{=} J(d, \phi)-I(d) .
$$

When there is one single constraint like for $Z_{L}(N)$, the large deviations expression can be obtained by saddle point techniques $[13,19]$. For more than one constraint it seems easier to work variationally. Let us introduce the cumulant generating function $h$ associated to $\pi^{\lambda}$,

$$
h(s, t) \stackrel{\text { def }}{=} \log \left[\sum_{n=0, \mu}^{\infty} \pi^{\lambda}(n, \mu) e^{s n+t \mu}\right],
$$

assuming by convention that the rate $\mu$ is zero in absence of client. After some computations (see [15] for details), we obtain $I$ and $J$ as Legendre transforms of the cumulant generating function, namely:

$$
\begin{aligned}
J(d, \phi) & =\frac{d}{1-d} \lambda_{n}(d, \phi)+\frac{\phi}{1-d} \lambda_{\mu}(d, \phi)-h\left(\lambda_{n}(d, \phi), \lambda_{\mu}(d, \phi)\right), \\
I(d) & =\frac{d}{1-d} \lambda_{n}^{\prime}(d)-h\left(\lambda_{n}^{\prime}(d), 0\right),
\end{aligned}
$$

with $\lambda_{n}(d, \phi)$ and $\lambda_{\mu}(d, \phi)$ the Lagrange multipliers, conjugate respectively to the density and flux, and solution of the equations

$$
\left\{\begin{array}{l}
\frac{\partial h}{\partial s}\left(\lambda_{n}(d, \phi), \lambda_{\mu}(d, \phi)\right)=\frac{d}{1-d} \\
\frac{\partial h}{\partial t}\left(\lambda_{n}(d, \phi), \lambda_{\mu}(d, \phi)\right)=\frac{\phi}{1-d} \\
\frac{\partial h}{\partial s}\left(\lambda_{n}^{\prime}(d), 0\right)=\frac{d}{1-d} .
\end{array}\right.
$$

The ordinary FD $\phi(d)$ is the minimizer of $K(\phi \mid d)$ and actually corresponds to 

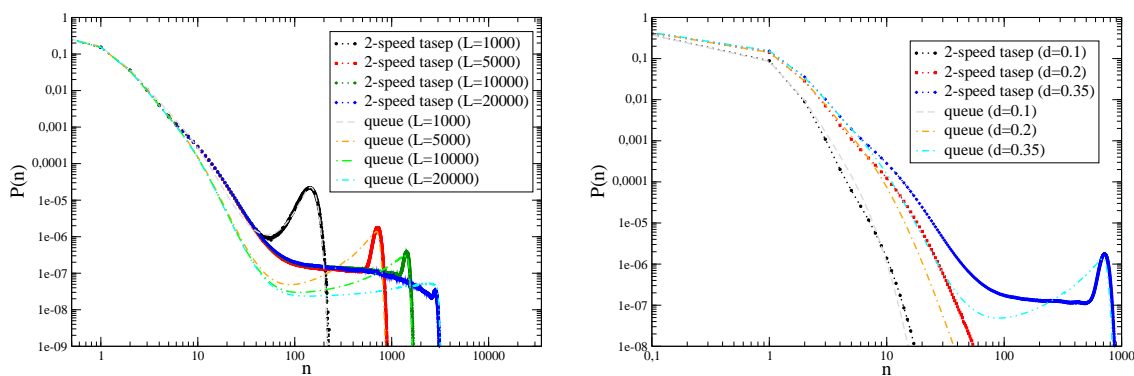

Fig. 2. Comparison of particle cluster vs queue's size distribution for set of parameters $\mu_{a}=10 \mu_{b}=100, \gamma=10$ and $\delta=1$ for various sizes $L$ with fixed density $d=0.35$ (left) and various densities with fixed size $L=5000$ (right), the number of queues being $(1-d) L$.

$$
K(\phi(d) \mid d)=0 .
$$

The small i.e. Gaussian fluctuations are then obtained by expanding $K$ at second order in $\phi-\phi(d)$. Denoting the dual Hessian,

$$
H^{\star}(s, t) \stackrel{\text { def }}{=}\left[\begin{array}{ll}
h_{s s} & h_{s t} \\
h_{t s} & h_{t t}
\end{array}\right],
$$

with the use of shorthand notations for the derivatives, representing the covariant matrix between the charges of the queues and the flux, we find the following expression for the variance of the FD:

$$
\operatorname{Var}(\phi \mid d)=\frac{(1-d)^{2}}{L}\left(H_{t t}^{\star-1}\right)^{-1}
$$

Under the canonical ensemble constraint, the single queue distribution can be obtained from the partition function as

$$
\begin{aligned}
p_{C E}(n, \mu)= & p_{\lambda}(n, \mu) \frac{Z_{L-1}(N-n)}{Z_{L}(N)} \\
\simeq p_{\lambda}(n, \mu) \exp L[ & h(s(d-x), 0)-h(s(d), 0) \\
& \left.\quad+\frac{d-x}{1-d-x} s(d-x)-\frac{d}{1-d} s(d)\right],
\end{aligned}
$$

with $x \stackrel{\text { def }}{=} n /(N+L)$ and the density constraint satisfied by $s(d)$ :

$$
\frac{\partial h}{\partial s}(s(d), 0)=\frac{d}{1-d},
$$

A direct comparison with the 2-speed TASEP of cluster size distributions is shown on Figure 2. The correspondance is rather accurate, in particular for the presence and location of the bumps signaling apparition of wide jams. In both cases, condensation is observed as a finite size effect, the bumps being replaced by a plateau at larger system size as seen on Figure 2.a. 

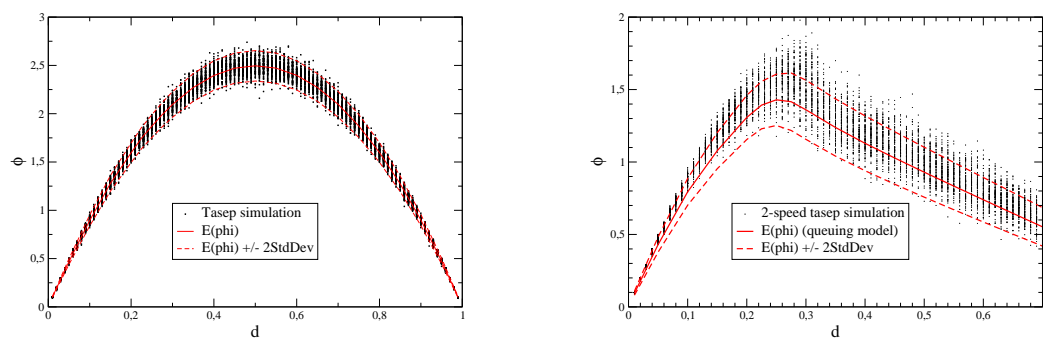

Fig. 3. Comparison between simulation for the simple TASEP FD (left) and the 2-speed TASEP with $\mu_{a}=10 \times \mu_{b}=10 \times \gamma=100 \times \delta$ (right) with small deviations results (two standard deviations) obtained from the queuing processes.

\subsection{Comparison with 2-speed TASEP}

As a sanity check, let us apply first this formula to the simple $M / M / 1$ queue corresponding to one single speed level $\left(\mu_{a}=\mu_{b}=\mu\right)$, i.e. a TASEP process. The rate of arrival $\lambda$ is set by convenience to $\lambda=d \mu$. The cumulant-generating function then reads

$$
h(s, t)=\log (1-d)+\log \frac{1+d e^{s}\left(e^{t v}-1\right)}{1-d e^{s}} .
$$

The FD rate function for TASEP then reads $(\bar{d} \stackrel{\text { def }}{=} 1-d)$

$$
K(\phi \mid d)=\frac{d}{\bar{d}} \log \frac{\mu d-\phi}{\mu d^{2}}+\frac{\phi}{\mu \bar{d}} \log \frac{\phi^{2}}{(\mu d-\phi)(\mu \bar{d}-\phi)}-\log \frac{\mu \bar{d}^{2}}{\mu \bar{d}-\phi},
$$

yielding an expression for FD and variance that matches direct computation [15]:

$$
\phi(d)=\mathbb{E}(\phi \mid d)=\mu d \bar{d}, \quad \operatorname{Var}(\phi \mid d)=\frac{\mu^{2}}{N+L} d^{2} \bar{d}^{2} .
$$

In the case of the model of Section 3, the assumption of independence between servers is not valid anymore, but corresponds to a mean-field approximation [15]. From (5), we get for the cumulant generating function

$$
h(s, t)=\log \left(\pi_{0}\left(1+\frac{\mu_{b} e^{\mu_{a} t}-\mu_{a} e^{\mu_{b} t}}{\mu_{a}-\mu_{b}}\right)+\frac{\left(\mu_{a}-\lambda e^{s}\right) e^{\mu_{b} t}+\left(\lambda e^{s}-\mu_{b}\right) e^{\mu_{a} t}}{\mu_{a}-\mu_{b}} g\left(e^{s}\right)\right),
$$

from which the Legendre transform as well as the Hessian $H^{*}\left(s^{\star}, 0\right)$ for the small fluctuations can be obtained, where $s^{\star}$ is the point which satisfies

$$
e^{s^{\star}} g^{\prime}\left(s^{\star}\right)=\frac{d}{1-d} .
$$

A comparison with the corresponding 2-speed TASEP is shown on Figure 3. The observed discrepancy can be traced back to various approximations made in the mean-field theory, in particular the assumption of independence between successive servers. This could be possibly cured by a refined mean field approach, replacing the product form ansatz with a Bethe approximation. This requires to solve exactly the 2-servers problem, but the large deviations approach would follow the same lines. 


\section{Acknowledgments}

This work was supported by the French National Research Agency (ANR) grant No ANR-08-SYSC-017.

\section{References}

1. M. Schreckenberg, A. Schadschneider, K. Nagel, and N. Ito. Discrete stochastic models for traffic flow. Phys. Rev., E51:2339, 1995.

2. B.S. Kerner. The Physics of Traffic. Springer Verlag, 2005.

3. Y. Sugiyama et al. Traffic jams without bottlenecks: experimental evidence for the physical mechanism of the formation of a jam. New Journal of Physics, 10:1-7, 2008.

4. K. Nagel and M. Schreckenberg. A cellular automaton model for freeway traffic. J. Phys. I,2, pages 2221-2229, 1992.

5. R. Barlović, L. Santen, A. Schadschneider, and M. Schreckenberg. Metastable states in cellular automata for traffic flow. Eur. Phys. J., B5:793, 1998.

6. M. Blank. Hysteresis phenomenon in deterministic traffic flows. Journal of Statistical Physics, 120:627-658, 2005.

7. C. Appert and L. Santen. Boundary induced phase transitions in driven lattice gases with meta-stable states. PRL, 86:2498, 2001.

8. T. M. Liggett. Interacting Particle Systems. Springer, Berlin, 2005.

9. F. Spitzer. Interaction of Markov processes. Adv. Math., 5:246, 1970.

10. M. Samsonov, C. Furtlehner, and J.-M. Lasgouttes. Exactly solvable stochastic processes for traffic modelling. Technical Report 7278, INRIA, 2010.

11. C. Furtlehner and J.-M. Lasgouttes. A queueing theory approach for a multispeed exclusion process. In Traffic and Granular Flow 'O7, pages 129-138, 2007.

12. J. Kaupuz̃s, R. Mahnke, and R. J. Harris. Zero-range model of traffic flow. Phys. Rev. E, 72:056125, 2005.

13. M. R. Evans, S. N. Majumdar, and R. K. P. Zia. Canonical analysis of condensation in factorized steady states. Journal of Statistical Physics, 123(2):357-390, 2006.

14. C.M. Harris. Queues with state-dependant stochastic service rate. Operation Research, 15:117-130, 1967.

15. C. Furtlehner, J.-M. Lasgouttes, and M. Samsonov. One-dimensional particle processes with acceleration/braking asymmetry. arXiv:1109.1761, 2010.

16. K. Nagel and M. Paczuski. Emergent traffic jams. Phys. Rev. E, 51(4):2909-2918, 1995.

17. F. P. Kelly. Reversibility and stochastic networks. John Wiley \& Sons Ltd., 1979. Wiley Series in Probability and Mathematical Statistics.

18. H. Touchette. The large deviation approach to statistical mechanics. Physics Reports, 478:1-69, 2009.

19. G. Fayolle and J.-M. Lasgouttes. Asymptotics and scalings for large closed product-form networks via the Central Limit Theorem. Markov Proc. Rel. Fields, 2(2):317-348, 1996. 